\title{
LA EVALUACIÓN FORMATIVA EN UNA SECUENCIA DE ENSEÑANZA APRENDIZAJE DE CIENCIAS EXPERIMENTALES
}

Formative assessment in a teaching-learning sequence of experimental sciences A avaliação formativa em uma sequência de ensino aprendizagem de ciências experimentais

Las autoras agradecen al proyecto EDU2015-64642-R (MINECO/FEDER) con financiación del Ministerio de Economía y Competitividad de España y el Fondo Europeo de Desarrollo Regional su colaboración.

\section{Vanessa Ortega-Quevedo 1 (1) \\ Noelia Santamaría-Cárdaba (2) \\ Cristina Gil Puente (3)}

(1) Universidad de Valladolid. vanessaortegaquevedo@gmail.com

(2) Universidad de Valladolid.noeliacardaba@hotmail.com

(3) Universidad de Valladolid. cgil@dce.uva.es

\begin{abstract}
Resumen
Este trabajo presenta una experiencia cuya finalidad es originar aprendizajes significativos a través de la aplicación de una SEA que toma como base la evaluación formativa. Dicha propuesta, se ha llevado a cabo en el área de Ciencias Naturales, concretamente en dos centros educativos de Segovia en $6^{\circ}$ curso de Educación Primaria. Los resultados obtenidos muestran que los estudiantes han mantenido una participación activa, la cual ha permitido a estos reconstruir sus aprendizajes a partir de las intervenciones realizadas por sus compañeros o por el propio maestro. En conclusión, a pesar de las dificultades encontradas se la propuesta ha resultado positiva y refleja que aplicar la evaluación formativa permite la creación de aprendizajes significativos.

Palabras clave: Evaluación Formativa; Pensamiento Crítico; Didáctica de las Ciencias Experimentales; Ciencias Naturales; Educación Primaria
\end{abstract}




\begin{abstract}
This project present an experience whose aim is originate meaningful learnings through the application of a Teaching-Learning Sequel' (SEA) that take as basis the formative assessment. This proposal has been put into practice in the area of Natural Sciences, specifically in an educational center in Segovia (Spain) in 6th grade Elementary Education. The results show that the students have maintained an active participation during which they have been able to reconstruct their learning from the interventions made by their classmates or by the teacher. To conclude, the proposal has been positive and reflects that to apply a formative assessment improve the meaningful learnings.
\end{abstract}

Keywords: Formative assessment; Critical Thinking; Didactics of Experimental Sciences; Natural Sciences; Elementary Education

\title{
Resumo
}

Este trabalho apresenta uma investigação cujo objetivo é verificar a importância da aprendizagens originou através da aplicação de uma SEA que toma como base a avaliação formativa. A referida proposta foi realizada na área de Ciências Naturais, concretamente, em dois centros de educação de Segóvia em $6^{\circ}$ curso de Ensino Primário. Os resultados conseguidos revelam que os estudantes mantiveram uma participação ativa, que lhes permitiu reconstruir a aprendizagem requerida a partir das intervenções realizadas pelos seus parceiros ou pelo próprio professor. Em conclusão, apesar das dificuldades encontradas a proposta obteve resultados positivos e revela que aplicar a avaliação formativa permite a criação de consideráveis índices de aprendizagem.

Palavras-chave: Avaliação Formativa; Pensamento Crítico; Didática das Ciências Experimentais; Ciências Naturais; Ensino Primário

\section{Introducción}

Esta experiencia entiende la evaluación formativa como "un proceso de evaluación cuya finalidad principal es mejorar los procesos de enseñanza-aprendizaje que tienen lugar” (López-Pastor, Monjas, Manrique, Barba \& González, 2008, p.464). En consecuencia, se defiende ésta como un proceso en el cual los docentes y los estudiantes mejoran 
gracias a las valoraciones que se producen durante el proceso de enseñanza-aprendizaje (Popham, 2013; López-Pastor, 2017).

Se ha escogido este enfoque porque permite evaluar la significatividad de los aprendizajes del alumnado, en relación con el desarrollo de su competencia científica. Asimismo, esta propuesta de evaluación se integra de un modo mimético en el proceso de enseñanza-aprendizaje, de forma que la evaluación es utilizada "como medio de perfeccionamiento sobre la marcha de la actividad didáctica” (Rosales, 1988, p.107).

Ahora bien, el modelo de evaluación formativa que se emplea es el comunicativo-crítico. Quinquer (2000) señala que esta orientación entiende el aprendizaje como la construcción del conocimiento realizada por un estudiante, da relevancia a la comunicación entre personas y destaca la necesidad de potenciar la autonomía de los discentes. Por tanto, este modelo implementa una evaluación basada en el diálogo y centrada en el desarrollo de competencias (López-Pastor, 2017). Durante este proceso, la acción evaluadora consiste en la conducción de argumentaciones hacia lo que los científicos establecen como concepciones aceptables sobre Ciencia, Tecnología y Sociedad (Acevedo \& García; 2016; Vázquez, Acevedo \& Manassero, 2004).

\section{Contextualización}

Las experiencias se han realizado en dos escuelas públicas de Segovia. Ambos centros cuentan con dos líneas en el curso donde se ha realizado la intervención ( $6^{\circ}$ de Educación Primaria). Las características de estos centros son:

- Centro 1: el nivel económico y cultural de las familias cuyos descendientes acoge el centro es medio-bajo. El porcentaje de alumnado de minorías étnicas e inmigrantes es muy elevado.

- Centro 2: en este caso, el nivel económico y cultural de las familias es algo más elevado que en el centro 1 (medio) y el porcentaje de estudiantes inmigrantes o pertenecientes a minorías es menor.

\section{Diseño y desarrollo}

La finalidad de esta experiencia es regular el aprendizaje en el desarrollo de una Secuencia de Enseñanza Aprendizaje (SEA). Esta se va a llevar a cabo en los dos grupos de los centros presentados y está formada por una SEA elaborada en base al 
denominado ciclo de aprendizaje $7 \mathrm{E}^{1}$ creado por el proyecto CYTPENCRI ${ }^{2}$. Dicha SEA (véase Tabla 1) está orientada tanto al desarrollo de habilidades de pensamiento crítico, como a la consecución de conocimientos básicos sobre la ciencia y la tecnología en relación con la obtención de energía.

Tabla 1.

Esquema del desarrollo de la SEA

\begin{tabular}{|c|c|c|}
\hline Sesiones & Actividades & Organización \\
\hline 1 & $\begin{array}{l}\text { Introducción } \\
\text { Rutina de pensamiento: Generar, clasificar conectar, elaborar. } \\
\text { Conocimientos previos } \\
\text { Puesta en común de las conclusiones generadas por las rutinas de } \\
\text { pensamiento. } \\
\text { Contenidos } \\
\text { Escuchan-preguntan: explicación del concepto de energía, sus formas de } \\
\text { manifestación, sus propiedades. } \\
\text { Procedimientos-Actitudes } \\
\text { Resolución de preguntas de reflexión a través del uso de procesos de } \\
\text { pensamiento crítico. } \\
\text { Consolidación } \\
\text { Puesta en común de las conclusiones de las preguntas de reflexión. }\end{array}$ & \\
\hline 2 & $\begin{array}{l}\text { Introducción } \\
\text { Rutina de pensamiento: Palabra, idea, frase. } \\
\text { Conocimientos previos } \\
\text { Igual-primera sesión. } \\
\text { Contenidos } \\
\text { Las energías renovables y no renovables, los impactos en el medio. } \\
\text { Procedimientos-Actitudes } \\
\text { Igual-primera sesión. } \\
\text { Consolidación } \\
\text { Igual-primera sesión. }\end{array}$ & $\begin{array}{l}\text { Parejas y } \\
\text { grupo-clase }\end{array}$ \\
\hline 3 & $\begin{array}{l}\text { Introducción } \\
\text { Rutina de pensamiento: Veo, pienso, me pregunto. } \\
\text { Conocimientos previos } \\
\text { Igual-primera sesión. } \\
\text { Contenidos } \\
\text { El método fracking. } \\
\text { Procedimientos-Actitudes } \\
\text { Igual-primera sesión. } \\
\text { Consolidación } \\
\text { Igual-primera sesión. }\end{array}$ & \\
\hline
\end{tabular}

El sistema de evaluación formativa que se ha diseñado con el fin de regular el aprendizaje en la SEA tiene como objetivo "la mejora de los procesos de aprendizaje del alumno, el perfeccionamiento del docente y los procesos de enseñanza-aprendizaje que tienen lugar en un contexto educativo” (López-Pastor, 2009, p. 94). En la Tabla 2 se recogen las técnicas e instrumentos que se van a utilizar para valorar los aprendizajes.

\footnotetext{
${ }^{1}$ El ciclo 7E está formado por 7 fases: Extraer, Enganchar, Explorar, Explicar, Elaborar, Extender y Evaluar.

${ }^{2}$ CYTPENCRI: Educación de las Competencias Científica, Tecnológica y Pensamiento Crítico Mediante la Enseñanza de Temas de Naturaleza de Ciencia y Tecnología. 
Tabla 2.

Técnicas e instrumentos de evaluación

\begin{tabular}{ll}
\hline \multicolumn{1}{c}{ Técnicas } & \multicolumn{1}{c}{ Instrumentos } \\
\hline Autoevaluación del alumnado & Ficha $^{3}$ \\
Observación participante & Diario del maestro \\
Autoevaluación docente & Tabla de autoevaluación $^{5}$ \\
\hline
\end{tabular}

\section{Evaluación}

En la tabla 3 se recogen los resultados derivados de la intervención respaldados por la triangulación de los instrumentos de evaluación.

Tabla 3.

Resultados de la intervención

Centro 1

Evaluación de las actividades a partir de la autoevaluación del alumnado y de la observación participante

Introducción previos

Contenidos

Procedimientos-

Actitudesconsolidación
Se encontraron dificultades para que el alumnado realizase los procesos cognitivos de las rutinas. Se solucionó invirtiendo más tiempo en la actividad y realizando preguntas de apoyo.

Algunos estudiantes no poseían los conceptos básicos. Se tuvo que dedicar tiempo a su compresión para poder iniciar su transferencia a otros contextos.

No se han podido trabajar en profundidad la trasferencia de todos los contenidos por las dificultades encontradas y la limitación temporal.

Los participantes no estaban acostumbrados a esta metodología y hubo que dedicar más tiempo a la realización de los debates.
Los procesos planteados se efectuaron correctamente.

Los discentes poseían una buena base previa.

Todos los contenidos se han trabajado adecuadamente.

El alumnado llevó a cabo las intervenciones

satisfactoriamente creando situaciones de aprendizaje.

Autoevaluación docente a partir de la tabla de autoevaluación y de la observación participante Resultados Se han tenido que realizar adaptaciones en tiempos y contenidos.

No se han llevado a cabo adaptaciones.

\section{Conclusiones}

Tras realizar esta propuesta, se concluye que durante las experiencias se han regulado los procesos de aprendizaje del alumnado hasta lograr aprendizajes significativos en ambos centros. Por consiguiente, se determina que la propuesta de evaluación formativa ha resultado exitosa y permite apreciar cómo el alumnado mejora su aprendizaje, el

\footnotetext{
${ }^{3}$ Soporte físico donde el alumnado deja constancia de la evolución de su aprendizaje.

${ }^{4}$ Instrumento para la descripción y el análisis de la realidad escolar.

${ }^{5}$ Instrumento de evaluación de las decisiones docentes tomadas a lo largo de las experiencias.

La evaluación formativa en una secuencia de enseñanza aprendizaje de Ciencias Experimentales
} 
docente mejora su práctica y cómo se perfeccionan los procesos de enseñanzaaprendizaje.

\section{Referencias}

Acevedo, J.A., \& García, A. (2016). Algo antiguo, algo nuevo, algo prestado. Tendencias sobre la naturaleza de la ciencia en la educación científica. Revista Eureka sobre Enseñanza y Divulgación de las Ciencias 13(1), 3-19. Doi: 10498/18010.

Cañal, P. (2012). La evaluación de la competencia científica requiere nuevas formas de evaluar los aprendizajes. En E. Pedrinaci (coord.). 11 ideas clave. El desarrollo de la competencia científica (pp.241-269). Barcelona: Graó.

López-Pastor, V. M., Monjas, R., Manrique, J. C., Barba, J. J., \& González, M. (2008). Implicaciones de la evaluación en los enfoques de educación cooperativa. El papel de la evaluación formativa y compartida en la necesaria búsqueda de coherencia. Cultura y Educación, 20(4), 457-477.

López-Pastor, V. M. (Coord.). (2009). Evaluación Formativa y Compartida en Educación Superior. Propuestas, técnicas, instrumentos y experiencias. Madrid: Narcea.

López-Pastor, V. M. (2017). Evaluación formativa y compartida: evaluar para aprender y la implicación del alumnado en los procesos de evaluación y aprendizaje. En V. M. López-Pastor y A. Pérez-Pueyo (coords.). Buenas prácticas docentes. Evaluación formativa y compartida en educación: experiencias de éxito en todas las etapas educativas. León: Universidad de León

Popham, W. J. (2013). Evaluación trans-formativa. El papel transformador de la evaluación formativa. Madrid: Narcea.

Quinquer, D. (2000). Modelos y enfoques sobre evaluación: el modelo comunicativo. En A. Parcerisa (Dir.), Evaluación como ayuda al aprendizaje (pp.13-20). Barcelona: Laboratorio Educativo; Graó.

Rosales, C. (1988). Criterios para una Evaluación Formativa. Objetivo. Contenido. Profesor. Aprendizaje. Recursos. Madrid: Narcea. 
Vázquez, A., Acevedo, J.A., Manassero, M.A. (2004). Consensos sobre la naturaleza de la ciencia: evidencias e implicaciones para su enseñanza. Revista Iberoamericana de Educación. Recuperado de: rieoei.org/deloslectores/702Vazquez.PDF 